\title{
Comparison of tuberculosis surveillance systems in low-incidence industrialised
} \section{countries}

\author{
Z. Mor* ${ }^{*}$, G.B. Migliori", S.P. Althomsons ${ }^{+, \S}$, R. Loddenkemper ${ }^{f}$, \\ L. Trnka** and M.F. lademarco ${ }^{+}$
}

ABSTRACT: The comparative analysis of National Tuberculosis Control Programmes (NTPs) in industrialised, low-tuberculosis-incidence countries is limited. Analysis of applied methods, function and accumulated experience contributes to improving global tuberculosis control.

A questionnaire addressing NTP surveillance infrastructure and characteristics was completed in 19 industrialised countries, with populations of $>3$ million and annual notified tuberculosis incidence rates of $<16$ cases per 100,000 population (2003 data).

All European countries surveyed adopted World Health Organization Collaborating Centre for the Surveillance of Tuberculosis in Europe (EuroTB) definitions. Surveillance information, which usually includes names, was transferred electronically to the national level in 17 out of the 19 countries. Surveillance systems capture process and social determinants. Case notification to the central level occurred within a median period of 7 days, independent of mandatory notification requirements. The mean completeness of tuberculosis case-reporting was estimated to be $93.5 \%$ (range 65-100\%). Integration between HIV and tuberculosis registries was performed in two countries, and, in seven others, both databases were cross-matched periodically.

National Tuberculosis Control Programme function in industrialised low-incidence countries utilises well-established infrastructure and relies upon centralised operations. Approaches are consistent with current World Health Organization surveillance recommendations. The present study lays collaborative groundwork for additional multinational analyses for the enhancement of global tuberculosis surveillance, which may assist policy-makers in countries moving from medium to low rates of incidence.

\section{KEYWORDS: Global health, National Tuberculosis Programme, surveillance, tuberculosis}

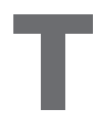
uberculosis (TB) control in industrialised countries varies substantially in its organisation, function and history. Consequently, it may be challenging to point to a set of discrete institutional components and label them the National Tuberculosis Control Programmes (NTPs). Each country has established NTP function, composed of an amalgamated network of organised public and private efforts, which has evolved in association with societal and economic trends in industrialised countries with what is now a low incidence of TB.

Surveillance performance, which provides notice of epidemiologically significant changes, is one of the fundamental public health activities necessary for the control and elimination of TB [1]. Since the 1950s, many countries have increasingly introduced organised surveillance activities at a national level. More recently, the World Health
Organization (WHO) began comprehensive worldwide annual reporting of traditional TB surveillance data, as well as elements of programme management, which also include treatment outcomes and drug supply [2, 3].

Although surveillance performance in industrialised countries has developed independent of supranational guidance, most are consistent with the current $\mathrm{WHO}$ recommendations [3]. The definitions used for surveillance have also been endorsed by the International Union Against Tuberculosis and Lung Disease [4]. Since the 1990s, substantial efforts have been invested at the international level in developing recommendations and guidance for specialised areas in countries with high TB rates and technical matters related to policy development, including transition issues in countries shifting from low to middle income or from high to medium rates of incidence [5-11].

\section{AFFILIATIONS}

*Public Health Services, Ministry of Health, Jerusalem, Israel. ${ }^{\#}$ Rollins School of Public Health, Emory University

${ }^{+}$Division of Tuberculosis Elimination, US Centers for Disease Control and Prevention (CDC).

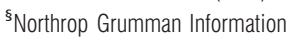
Technology, CDC Programs, Atlanta, GA, USA.

"WHO Collaborating Centre for TB and Lung Diseases, S. Maugeri Foundation, Care and Research Institute, Tradate, Italy

${ }^{f}$ German Central Committee against Tuberculosis, Berlin, Germany.

**National TB Surveillance Unit, Bulovka Hospital, Prague, Czech Republic.

CORRESPONDENCE

M.F. lademarco

Fax: 8448505028

E-mail: iademarcoMF@state.gov

Received:

March 192008

Accepted after revision:

July 242008

SUPPORT STATEMENT

The contents of the present article are solely the responsibility of the authors and do not necessarily represent the official views of parent organisations, including the Centers for Disease Control and Prevention (Atlanta, GA, USA).

STATEMENT OF INTEREST None declared.

Online ISSN 1399-3003 
TB incidence in most industrialised countries is low (defined by the WHO as $<20$ cases per 100,000 population [12, 13]). In addition to their developed economies and smaller populations, industrialised countries tend to have a highly functioning NTP in the setting of lower endemicity of TB. This occurs, in part, due to a combination of robust societal support of the NTP-associated agencies, consistent application of technologies and long-lasting control efforts. The essential elements of TB control in developed and low-incidence countries were addressed in Wolfheze Workshops [12] and published in the frameworks for TB control in Europe [13, 14].

The WHO annual global reports are a useful source for comparing countries' burden of disease and the accomplishments of the various NTPs [3]. However, this information does not fully address the existing variation between the different programmes, nor does it readily permit robust comparison between important components of NTPs. Thus a more detailed description and analysis of surveillance systems from industrialised countries should provide better understanding of operational standards and methods, based on decades of experience gained. Lessons learned could be used to contribute to the development of guidelines for both developing countries and countries undergoing transitions in TB incidence and economic status.

The present first descriptive study compares surveillance system function across industrialised countries with low TB incidence and lays the collaborative groundwork for advanced and additional analyses.

\section{METHODS}

Countries were chosen for the present study on the basis of: 1) high income, 2) low TB incidence, and 3) population of $>3$ million (to exclude city states and micronations). A highincome country was defined as having a gross national income of $>$ US $\$ 10,726$ per capita, as defined by the World Bank [15]. Low incidence was defined, for the purpose of the present study, as a mean of $<16$ new cases per 100,000 population annually during the period 2000-2003 [16]. This is a slightly lower threshold than the WHO standard of 20 new cases per 100,000 population, and includes those countries with a similar pattern of epidemiology, e.g. high incidence among foreignborn population. United Nations 2005 data were used for the total population size in each country [17].

NTP managers $(n=21)$ from the 19 eligible countries were contacted by electronic mail and asked to participate by completing a survey containing 48 questions in March 2006. The questionnaire focused on the notification process and the capacity of the surveillance system, such as reporting regulations, features of the data collection systems, time required for notification, periodic data analysis, process determinants (e.g. follow-up sputum culture results and records of adverse events due to treatment) and whether the system captured social determinants (e.g. country of origin, immigration date and status, homelessness, incarceration, marital status and occupation) [18]. Participants were asked whether incentives were provided to the reporting professionals (e.g. monetary value or access to the data) or whether penalties were instituted (e.g. civil litigation or reprimands by the medical regulatory authority). Parametric values were compared using the two-tailed Pearson's correlation test and continuous values were compared using a two-tailed unpaired t-test.

\section{RESULTS}

Completed questionnaires were received from all 19 countries between June and August 2006. All of the countries, except for Australia, Canada, New Zealand and the USA, form part of the European region of the WHO.

All 15 of the 15 European countries surveyed had adopted the WHO collaborating Centre for the Surveillance of Tuberculosis in Europe (EuroTB) case-reporting definitions and outcome categories (table 1) [19], which include a minimum set of variables required for notification, links between physician and laboratory notification systems, and unified outcome measures, promulgated in 2002 [13]. Data were submitted electronically in most of the 19 countries $(n=17 ; 89 \%)$ and sent to the national level in $16(84 \%)$ countries. Patients' data (name, address and identification number, when available) were reported in $10(53 \%)$ countries. France and Norway were the only two countries with mandatory national reporting of latent TB infection, although reporting was mandatory in Norway only if preventive treatment was started. TB suspects were also reported in 14 (74\%) countries; five countries reported suspects only as high as the local level, and nine countries reported to the national level. At the national level, 10 (50\%) countries captured process determinants and 12 (63\%) included social determinants.

In almost all countries, physicians and laboratories were required by law to report $\mathrm{TB}$ cases to a central authority, typically to the national level. However, in practice, nurses also participated in notifying cases (table 2). Ten (53\%) countries had penalties against professionals who failed to report cases, which were rarely, if ever, enforced. Ireland and the UK provided direct monetary incentives to professionals who notified public health officials of TB cases.

Although seven (37\%) countries did not specify a time requirement for reporting, the others required compulsory reporting 1-7 days following the date of TB diagnosis. Notification was reported to occur within 1-21 days (median 7 days), most notifications occurring later than required by law. No significant difference was found in reporting time among countries who required notification within a specified time period and those who had no time requirement $(r=0.28$; $\mathrm{p}=0.23)$. Eleven $(58 \%)$ countries indicated that each case was reported to the national level at the time of diagnosis. In the other eight countries, notification was conducted in a batched mode, mostly on a monthly basis. In Belgium, France and the $\mathrm{UK}$, reports were sent to the national level annually.

Verification of records was performed in all countries, mostly by comparing the national reporting form with laboratory notifications. Ten $(53 \%)$ countries operated with greater autonomy at the local level in data handling (i.e. decisions about recurrent cases and recording therapy compliance).

The electronic system that stored the data was independent in 10 $(53 \%)$ of the countries and integrated with other electronic reporting systems in the others (table 3). Laboratory results were integral to national control systems in all countries, and were required to be reported for TB cases in most countries ( $n=16 ; 84 \%)$. 


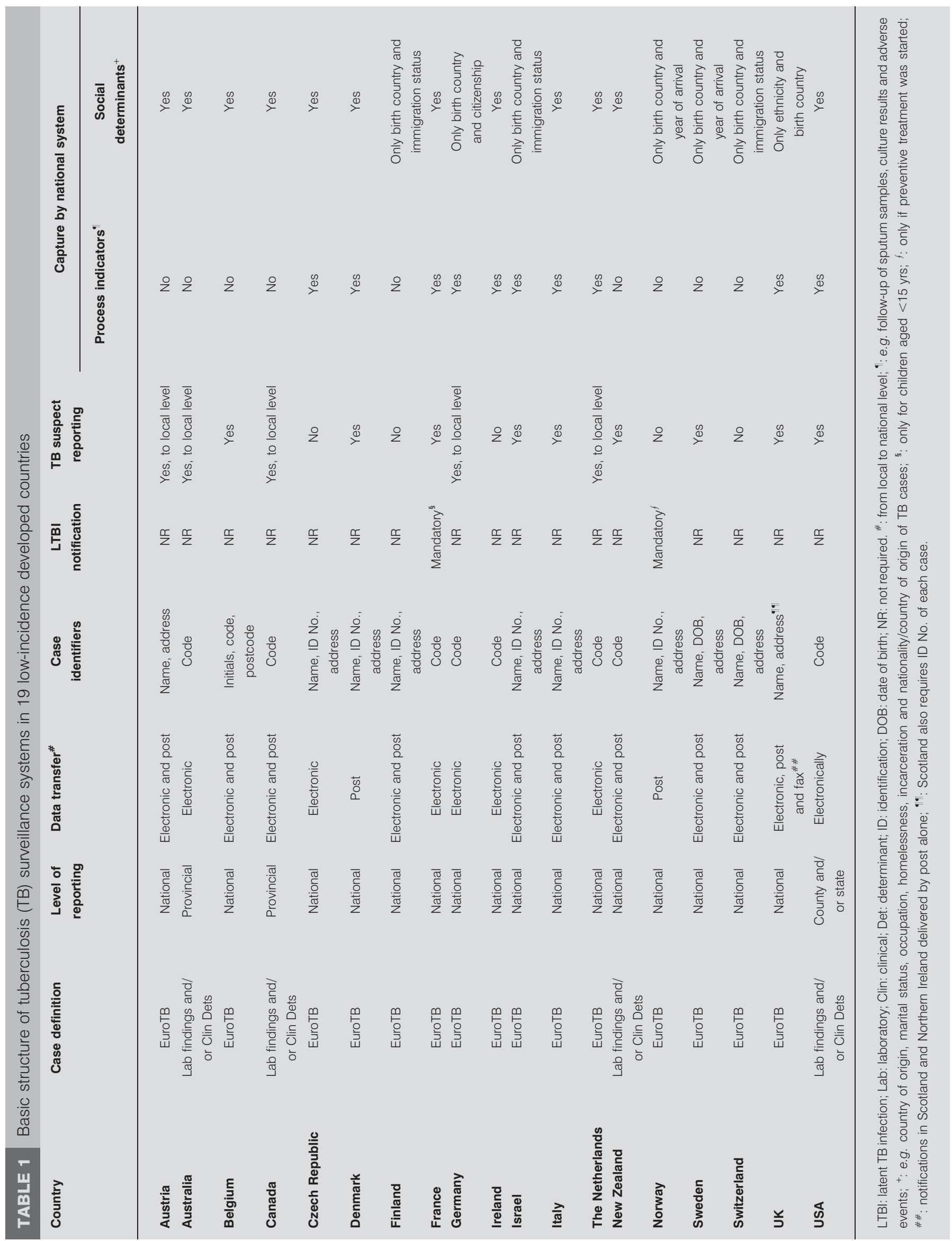




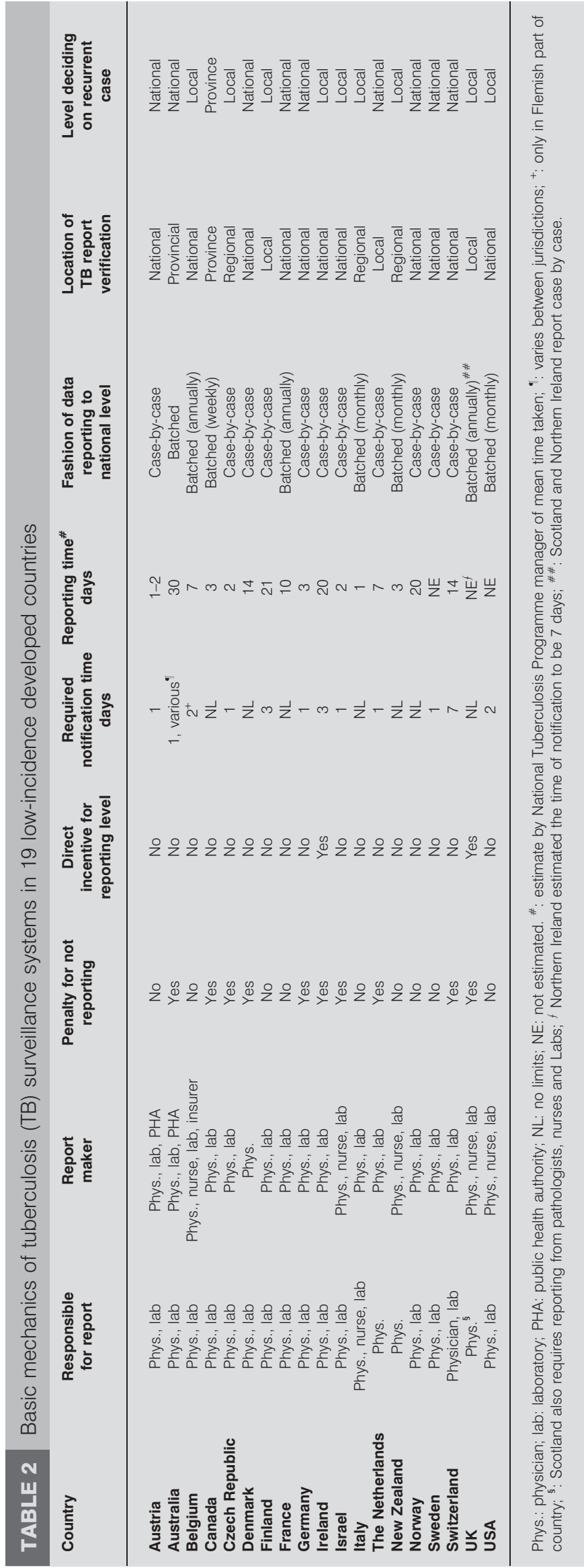

Treatment outcomes were included in all but four of the countries (Finland, France, Italy and Switzerland). TB surveillance systems included both surveillance and casemanagement-related data fields in most countries $(n=17$; $89 \%$ ), which include process indicators, such as treatment outcomes. Only Ireland, Italy and Switzerland restricted their system to traditional surveillance alone, limited to case counts and associated data. Data in most countries (17 out of 19) were analysed and reports disseminated annually.

Eight countries (the Czech Republic, Finland, France, Israel, New Zealand, Norway, the Netherlands and the USA) had validated TB case-reporting in formal studies and published their findings [20-22]. The completeness of TB case reports was estimated by NTP managers to range $65-100 \%$ (mean $93.5 \%$ ); only moderate correlation was found between estimated TB reporting completeness and the existence of penalties or incentives $(r=0.46 ; \mathrm{p}=0.048)$.

All countries registered drug susceptibility testing results (table 4). Although molecular laboratory methods were used in all countries, such results were required in only three (Austria, Denmark and Norway). Although HIV data were collected in each country, integration was performed automatically in only two (Denmark and Finland), whereas, in seven countries, TB and HIV databases were cross-matched periodically.

\section{DISCUSSION}

NTP surveillance function in the low-incidence industrialised countries surveyed is well established at both the national and subnational level, and is similar in aspects of reporting indicators. The flow of data moves in a prompt manner, even in those countries in which no time requirements are specified, no penalties are imposed and no incentives are provided. The data and information structure of the national registries in each country are consistent with WHO recommendations.

Data are transmitted electronically in most industrialised countries examined, and, in the others, systems are being upgraded to include electronic transmission, away from letters and faxes. As industrialised low-incidence countries continually increase and refine their use of information technology, data and information transfer among countries could be enhanced. This, for example, might help improve international coordination of immigrant health screening. Moreover, this surveillance enhancement could serve as a model for global surveillance system integration, monitoring additional communicable and emerging infections.

As the trend of global migration of people from countries with a high to those with a low incidence of TB increases [23], interest in international comparison of social characteristics may increase and benefit from a more standardised approach [24]. These particular data are used in case management and programme planning, including identification of high-risk groups, which underscore the continued association between morbidity and the social determinants of TB in industrialised countries [25]. A significant proportion of TB cases in industrialised countries are foreign-born individuals [24, 26].

NTP programme structure differs. For example, some countries use codes assigned to TB cases rather than personal identifiers for reporting, as required by law to protect 
confidentiality. Personal identifiers may facilitate internal reliability, whereas coding may promote notification since patients are ensured of their privacy protection. The ability to protect patients' rights and ensure data integrity is a delicate balance that countries are careful to maintain.

Minor differences exist in outcome definitions of TB and in reporting time among countries outside of the European region of the $\mathrm{WHO}$, which may limit comparison of these determinants among different NTPs [27]. In order to perform more accurate global comparisons, industrialised countries should consider further refinements and harmonisation of these definitions [28].
Nurses facilitate the reporting of TB cases in many of the countries, although only physicians and laboratories are obliged by law to notify to the national level. As nurses are increasingly becoming the backbone of human resources in public health systems, along with the increasing accreditation in their profession, further evaluation should be performed to assess whether formal transfer of some surveillance tasks from physicians to nurses could enhance reporting efficiency, completeness and quality.

Most TB control measures are performed at the provincial or regional level. Based on unsolicited comments from some

TABLE 3 Functional characteristics of tuberculosis (TB) surveillance systems in 19 low-incidence developed countries

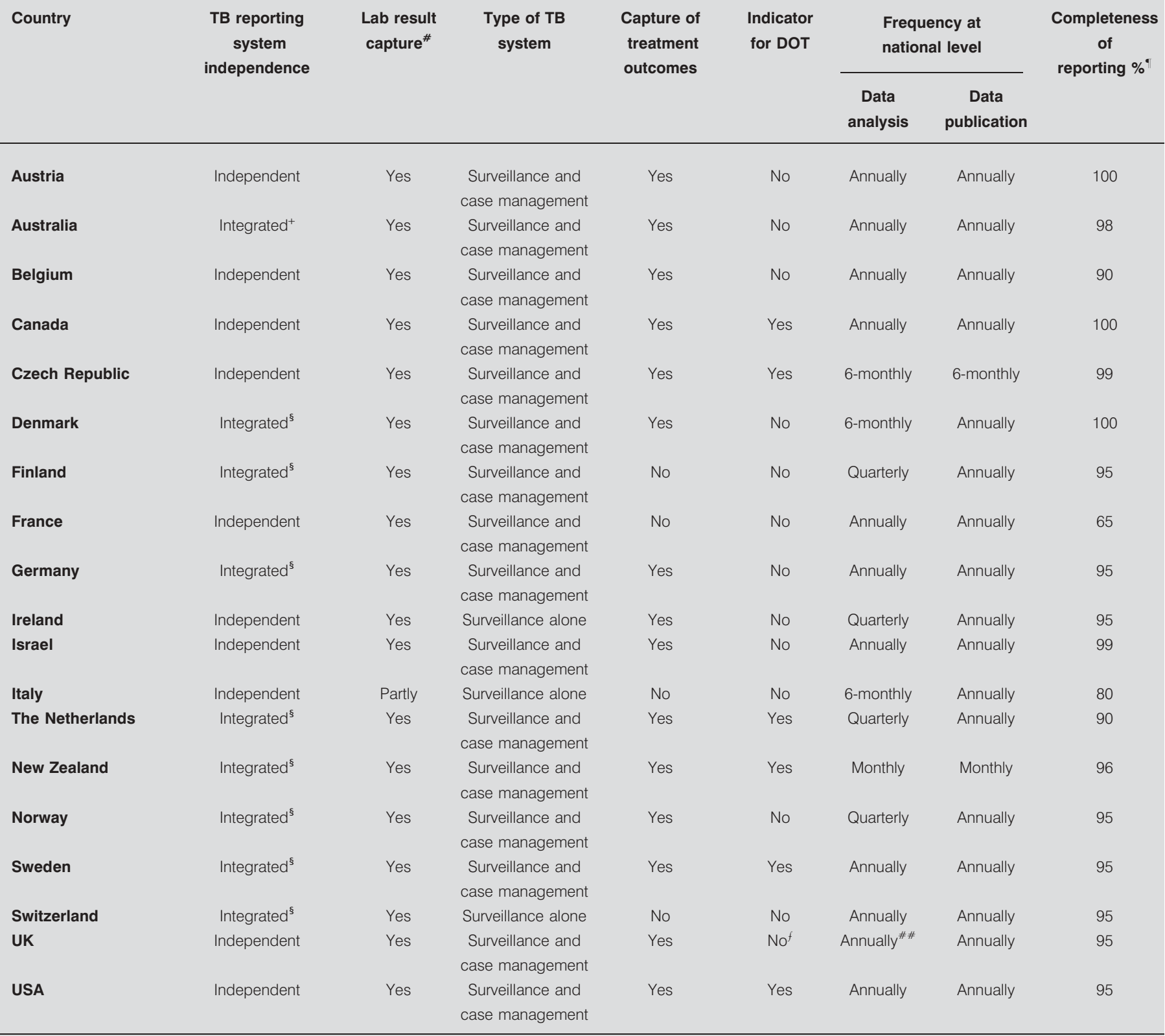

Lab: laboratory; DOT: directly observed therapy. \#: by national system, e.g. drug susceptibility testing and restriction fragment length polymorphism results; ": National

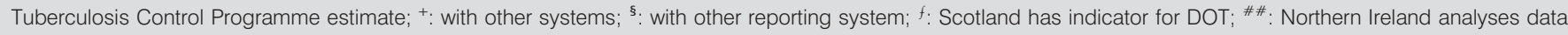
quarterly. 
countries, such as Australia, Belgium, Canada, Switzerland and the UK, significant intra-national differences were found among internal regions (e.g. states, cantons and provinces). These differences reflect greater autonomy or political constraints below the national level, which may limit standardisation of TB control. In order to increase the quality of data collection, collaborative efforts made by local professionals in internally diverse countries could increase internal reporting.

HIV infection status is an integral component of TB surveillance systems in only a few countries, an unexpected finding given the inextricable link between the two infections and, in many European countries, their association with immigration [24, 29]. Instead, HIV is captured in a separate registry, to which TB data are cross-matched in some countries. The reasons for separate registries may be to better protect confidentiality, minimise technical obstacles in integration, and ameliorate political and financial challenges. Additional studies should be preformed to assess whether HIV testing for each TB patient, strengthening TB/HIV monitoring [5] and merging the two databases for routine analysis improve the effectiveness of surveillance and patient care in industrialised countries. Surveys and special studies might be used in countries in which the merging of TB and HIV databases cannot be carried out for confidentiality reasons, or in which culture and drug susceptibility testing data are not collected for all individuals, to monitor the incidence and trends of multidrug- or extensively drug-resistant strains.
Finally, although incorporation of latent TB infection cases into the central registry and preventive treatment outcome indicators may further support the national TB registry, especially in countries which are close to TB elimination in their native-born population [13], no consensus was evident among the countries surveyed regarding the applicability and sustainability of their inclusion; further studies are needed in this area.

The specific NTP infrastructure of each country has a direct impact upon TB detection rates, treatment outcome and control [14]. Several factors may be operative. First, each currently industrialised country established its programme according to its own distinctive epidemiology, health infrastructure, political commitment, social norms, geographic structure and resources available. In most industrialised countries examined, NTP function was established independently, and when TB rates and economic status were different. Secondly, since TB epidemiology in industrialised countries is sensitive to immigration, different adjunctive components (including surveillance) were added to control programmes in some countries to address evolving local immigration patterns and to meet domestic naturalisation regulations. Finally, the unique organisation and funding of health systems in each country reflect individual development. Collectively, these factors underlying different NTP infrastructure make the development of cohesive international guidelines that are applicable to emerging industrialised countries challenging [30].

TABLE 4 Characteristics of laboratory tuberculosis (TB) and HIV data from Tuberculosis (TB) surveillance systems in 19 lowincidence developed countries

\begin{tabular}{|c|c|c|c|c|c|c|}
\hline Country & $\begin{array}{l}\text { DST required } \\
\text { for each case }\end{array}$ & $\begin{array}{l}\text { RFLP required } \\
\text { for each } \\
\text { case }\end{array}$ & $\begin{array}{l}\text { HIV data } \\
\text { collected } \\
\text { nationally }\end{array}$ & $\begin{array}{c}\text { HIV data automatically } \\
\text { integrated with } \\
\text { national TB system }\end{array}$ & $\begin{array}{c}\text { TB system } \\
\text { indicates HIV } \\
\text { infection }\end{array}$ & Source of HIV data \\
\hline
\end{tabular}

\begin{tabular}{|c|c|c|c|c|c|c|}
\hline Austria & Yes & Yes & Yes & No & No & \\
\hline Australia & Yes & No & Yes & No & Yes & TB cases tested for HIV \\
\hline Belgium & Yes & No & Yes & No & Yes & TB cases tested for HIV \\
\hline Canada & Yes $^{\#}$ & No & Yes & No & Yes & TB cases tested for HIV \\
\hline Czech Republic & Yes & No & Yes & No & No & \\
\hline Denmark & Yes & Yes & Yes & Yes (AIDS only) & Yes & Databases cross-matched \\
\hline Finland & Yes & No & Yes & Yes & Yes & Databases cross-matched \\
\hline France & Yes & No & Yes & No & No & \\
\hline Germany & Yes & No & Yes & No & No & \\
\hline Ireland & Yes & No & Yes & No & Yes & TB cases tested for HIV \\
\hline Israel & Yes & No & Yes & No & Yes & Databases cross-matched \\
\hline Italy & No & No & Yes & No & Yes & Databases cross-matched \\
\hline The Netherlands & Yes & No & Yes & No & Yes & $\begin{array}{c}\text { Selected TB cases tested } \\
\text { for HIV }\end{array}$ \\
\hline New Zealand & Yes & No & Yes & No & Yes & Databases cross-matched \\
\hline Norway & Yes & Yes & Yes & No & No & Limited data \\
\hline Sweden & Yes & No & Yes & No & No & \\
\hline Switzerland & Yes & No & Yes & No & No & \\
\hline UK & Yes & No & Yes & No & Yes & Databases cross-matched \\
\hline USA & Yes & No & Yes & No & Yes & Databases cross-matched \\
\hline
\end{tabular}

DST: Drug susceptibility testing; RFLP: restriction fragment length polymorphism. ${ }^{*}$ : although not legally required, in practice, DST is performed for all new isolates upon the physician's request; ' : with the exception of Scotland. 
Effective linkage between laboratories and public health authorities, especially electronic, is helpful for ensuring completeness of reporting and increasing the validity of the national registry [22]. Laboratories that confirm TB cases have been considered the most complete source of data [20,31]. It was therefore expected that greater completeness of reporting would be found among countries in which reporting by the laboratory was required; however, this could not be demonstrated as it applied to only two countries in the present study. Moreover, there may be under-reporting among nonlaboratory-confirmed cases, where the diagnosis is based on clinical findings alone.

Global TB figures are reported annually by the WHO and compare incidence across countries. It would be worthwhile to evaluate whether harmonised approaches to programme evaluation might further encourage periodic inter-country evaluation of the completeness and validity of TB surveillance. Validation of surveillance data is often costly and labour intensive [22], yet is the basis for estimating case detection rates $[25,32]$. Although intuitively true, there is no evidence that decentralisation of TB surveillance system operations and function strengthens overall information quality.

The present survey results may further assist countries with a higher TB incidence in improving their surveillance systems. For example, the present authors believe that the use of a reliable nationwide electronically connected system that includes the national level should also be established between TB laboratories and the national HIV/AIDS registry. Moreover, reporting should include clinical, diagnostic and social determinants of the patients and be registered at the national level. Greater autonomy of mid-level health departments may improve the verification of cases and the completion of missing information prior to transmission to the national level. Importantly, nurses should participate in data reporting, since they have the clinical experience and administrative skill to perform these tasks. Finally, evaluation of the completeness of the TB database and time required for cases to be reported should be performed periodically. In the present study, no significant association was found between incentives or penalties for the reporter and estimated completeness, and the present authors believe that it is the NTP manager's responsibility to persuade local professionals of the importance of notification, in part to decrease reporting bias.

The present study is subject to several limitations. First, it is cross-sectional and does not dissect the development of the various systems over time. Secondly, it excludes industrialised countries not meeting the present case definition, potentially missing extensive experience gained in TB control among other less-populated industrialised countries. Thirdly, the present survey was not designed to incorporate any measure of effectiveness, and thus it is not possible to prioritise or suggest how a difference in one system might affect another if applied. Finally, the present study, in its focus on surveillance systems, may miss other effects of NTP function and organisation that affect surveillance, such as changes in the quality of human resources and adherence to treatment over time. In order to obtain a deeper perspective of the effects of the structure of different NTPs, additional detailed comparisons should be performed, evaluating financial incentives, treatment funding, relationships between public and private providers, and additional structure indicators, such as qualification and training of personnel, and number and location of treatment sites.

\section{CONCLUSION}

National Tuberculosis Control Programme surveillance function in low-incidence high-income countries is well established, centrally operated and consistent with World Health Organization (including World Health Organization Collaborating Centre for the Surveillance of Tuberculosis in Europe) and International Union Against Tuberculosis and Lung Disease recommendations for reporting and data dissemination. Improved global harmonisation in outcome determinants and Internet-based electronic contact among various industrialised countries may enhance global tuberculosis control. Analysis of surveillance data and function may assist medium-incidence countries moving from medium to low rates of incidence and from vertically to horizontally organised healthcare systems. Nevertheless, detailed studies should be performed to compare the structure, process and effectiveness of different National Tuberculosis Control Programme systems in order to identify the fundamental attributes of an optimal system.

\section{ACKNOWLEDGEMENTS}

The authors wish to thank all of the National Tuberculosis Control Programme managers and the technical staff in the various countries approached, as follows: C. Hain (Federal Ministry for Health and Women, Vienna, Austria); V. Krause, P. Roche and C. Walker (Dep of Health and Ageing, Canberra, Australia); M. Wanlin (Foundation against Respiratory Ailments and for Health Education, Brussels, Belgium); E. Ellis and D. Scholten (Public Health Agency of Canada, Ottawa, ON, Canada); J. Wallenfels (National TB Surveillance Unit, Prague, Czech Republic); P.H.S. Andersen (Statens Serum Institute, Copenhagen, Denmark); P. Ruutu (National Public Health Institute, Helsinki, Finland); D. Che and D. Antoine (Institute for Public Health Surveillance, Saint-Maurice cedex, France); B. Brodhun, W. Haas and B. Hauer (Robert Koch Institute, Berlin, Germany); J. O'Donnell (National Disease Surveillance Centre, Dublin, Ireland); M.G. Pompa (Ministry of Health, Rome, Italy); A. Leventhal (Public Health Services, Jerusalem, Israel); K. Abu Rumman (Ministry of Health, Amman, Jordan); I. Hamilton and A. Roberts (Public Health Directorate, Wellington, New Zealand); B.A. Winje (Norwegian Institute of Public Health, Oslo, Norway); V. Romanus (Swedish Institute for Infectious Disease Control, Stockholm, Sweden); C. Erkens and V. Kuyvenhoven (Royal Netherlands Chemical Society, The Hague, the Netherlands); J. Watson, J. Crofts and M. Kruijshaar (Health Protection Agency, London, UK); B. Smyth (Communicable Disease Surveillance Centre for Northern Ireland, Belfast, UK); J. McMenamin and A. Jackson (Health Protection Scotland, Glasgow, UK); and R. Salmon (Communicable Disease Surveillance Centre, Cardiff, UK).

The authors also wish to thank P. Helbling (Federal Office of Public Health, Berne, Switzerland), P. Lobue, T. Navin and V. Robison (all from the Division of Tuberculosis Elimination, Centers for Disease Control and Prevention, Atlanta, GA, USA), and the two anonymous reviewers at the European Respiratory Journal for their thoughtful constructive suggestions. Furthermore, the authors wish to thank D. Anh, P. van Maaren and P. Glaziou (World Health Organization Western 
Pacific Regional Office, Manila, the Philippines) for organising technical sessions for intermediate-burden countries and participating in various intellectual discussions, which served to inspire the concept behind this paper.

\section{REFERENCES}

1 Castro KG. Tuberculosis surveillance: data for decision making. Clin Infect Dis 2007; 44: 1268-1270.

2 World Health Organization WHO Tuberculosis Programme: Framework for Effective Tuberculosis Control. WHO/TB/ 94.179. Geneva, World Health Organization, 1994.

3 World Health Organization. Global Tuberculosis Control Surveillance, Planning, Financing. WHO Report 2007. WHO/ HTM/TB/2007.376. Geneva, World Health Organization, 2007.

4 Rieder HL, Watson JM, Raviglione MC, et al. Surveillance of tuberculosis in Europe. Recommendations of a Working Group of the World Health Organization (WHO) and the European Region of the International Union Against Tuberculosis and Lung Disease (IUATLD) for uniform reporting on tuberculosis cases. Eur Respir J 1996; 9: 1097-1104.

5 World Health Organization. Management of Collaborative TB/HIV Activities: Training for Managers at the National and Subnational Level. WHO/HTM/TB/2005.359a,b,c. Geneva, World Health Organization, 2005.

6 World Health Organization. Interim Recommendations for the Surveillance of Drug Resistance in Tuberculosis. WHO/ HTM/TB/2007.385. Geneva, World Health Organization, 2007.

7 World Health Organization. Engaging all Health Care Providers in TB Control: Guidance on Implementing Public-Private Mix Approaches. WHO/HTM/TB/ 2006.360. Geneva, World Health Organization, 2006.

8 World Health Organization. A Research Agenda for Childhood Tuberculosis. WHO/HTM/TB/2007.381. Geneva, World Health Organization, 2007.

9 World Health Organization. Advocacy, Communication and Social Mobilization to Fight TB. A Ten-Year Framework for Action. WHO/HTM/STB/2006.37. Geneva, World Health Organization, 2006.

10 World Health Organization. Empowerment of Tuberculosis Patients in Tuberculosis Control. WHO/HTM/ TB/2007.39. Geneva, World Health Organization, 2007.

11 World Health Organization Regional Office for the Western Pacific. Progress in countries with an intermediate burden of tuberculosis (IBC). In: Fifth Technical Advisory Group Meeting to STOP TB in the Western Pacific Region, Busan, Republic of Korea, March 15-18. Report series number RS/ 2006/GE/03(KOR). Manila, World Health Organization Regional Office for the Western Pacific, 2006; pp. 35-37. www.wpro.who.int/NR/rdonlyres/3465E02F-9DF8-48C6992F-DE357F9B99B0/0/5th_TAG_200605_FINAL.pdf Date last updated: November 2006. Date last accessed: February 28, 2006.

12 Clancy L, Rieder HL, Enarson DA, Spinaci S. Tuberculosis elimination in the countries of Europe and other industrialized countries. Eur Respir J 1991; 4: 1288-1295.

13 Broekmans JF, Migliori GB, Rieder HL, et al. European framework for tuberculosis control and elimination in countries with low incidence. Recommendations of a Working Group of the World Health organization
(WHO), International Union against Tuberculosis and Lung Disease (IUATLD) and Royal Netherlands Tuberculosis Association (KNCV) Working Group. Eur Respir J 2002; 19: 765-775.

14 Taylor Z, Nolan CM, Blumberg HM. Controlling tuberculosis in the United States. Recommendation from the American Thoracic Society, CDC, and the Infectious Diseases Society of America. MMWR Recomm Rep 2005; 54: 1-81.

15 World Bank, Data \& Statistics. http://go.worldbank.org/ D7SN0B8YU0 Date last accessed: February 28, 2006.

16 World Health Organization, Global Health Atlas. www. who.int/globalatlas/predefinedReports/default.asp. Date last accessed: February 28, 2006.

17 United Nations, World Population Prospects: the 2006 Revision Population Database. http://esa.un.org/unpp Date last updated: September 20, 2007. Date last accessed: October 1, 2007.

18 Mor Z, Migliori GB, Althomsons SP, Loddenkemper R, Trnka L, Iademarco MF. A Designated Questionnaire Comparing TB Surveillance Systems in Low-Incidence and High Income Countries. www.health.gov.il/mor/ tb_questioinnaire.dot Date last updated: September 14, 2006. Date last accessed: June 2, 2008.

19 Veen J, Raviglione M, Rieder HL, et al. Standardized tuberculosis treatment outcome monitoring in Europe. Recommendations of a Working Group of the World Health Organization (WHO) and the European Region of the International Union Against Tuberculosis and Lung Disease (IUATLD) for uniform reporting by cohort analysis of treatment outcome in tuberculosis patients. Eur Respir J 1998; 12: 505-510.

20 Trepka MJ, Beyer TO, Proctor ME, Davis JP. An evaluation of the completeness of tuberculosis case reporting using hospital billing and laboratory data; Wisconsin, 1995. Ann Epidemiol 1999; 9: 419-423.

21 Curtis AB, McCray E, McKenna M, Onorato IM. Completeness and timeliness of tuberculosis case reporting: a multistate study. Am J Prev Med 2001; 20: 108-112.

22 Migliori GB, Spanevello A, Ballardini L, et al. Validation of the surveillance system for new cases in a province of Northern Italy. Eur Respir J 1995; 8: 1252-1258.

23 International Organization for Migration, International Organization for Migration. www.iom.int Date last updated: June 17, 2007. Date last accessed: September 11, 2007.

24 Falzon D, Ait-Belghiti F. What is tuberculosis surveillance in the European Union telling us? Clin Infect Dis 2007; 44: 1261-1267.

25 Holtgrave DR, Crosby RA. Social determinants of tuberculosis case rates in the Unites States. Am J Prev Med 2004; 26: 159-162.

26 Farge D, Porcher R, Antoun F, et al. Tuberculosis in European cities: establishment of a patient monitoring system over 10 years in Paris, France. Int J Tuberc Lung Dis 2007; 11: 992-998.

27 Falzon D, Schoten J, Infuso A. Tuberculosis outcome monitoring - is it time to update European recommendations? Euro Surveill 2006; 11: 20-25.

28 Ditah IC, Reacher M, Palmer C, et al., Monitoring tuberculosis treatment outcome: analysis of national surveillance data from a clinical perspective. Thorax 2008; 63: 440-446. 
29 Joint United Nations Programme on HIV/AIDS, World Health Organization, 07 AIDS Epidemic Update. UNAIDS/ 07.27E/JC1322E. Geneva, Joint United Nations Programme on HIV/AIDS, 2007.

30 World Health Organization. WHO Tuberculosis Programme Framework for Effective Tuberculosis Control. WHO/TB/ 94.1:1-7. Geneva, World Health Organization, 1994.
31 Effler P, Ching-Lee M, Bogard A, Ieong M, Nekomoto T, Jernigan D. Statewide system of electronic notifiable disease reporting from clinical laboratories. JAMA 1999; 282: $1845-1850$

32 Lonnroth K, Uplekar M, Arora VK, et al. Public-private mix for DOTS implementation: what makes it work? Bull World Health Organ 2004; 82: 580-586. 